\title{
Cloud2IR an infrared and environmental SHM information system
}

\author{
by A. Crinière*, J. Dumoulin*,**, L. Mevel* and G. Andrade-Barroso* \\ * Inria, I4S Team, Campus de Beaulieu, 35042 Rennes cedex, France - antoine.criniere@inria.fr \\ ** LUNAM Université, IFSTTAR, COSYS, SII, F-44340 Bouguenais, France
}

\begin{abstract}
Cloud2IR is an autonomous software architecture, allowing multi-sensor connection (i.e. Infrared Thermography), dedicated to the long term monitoring of infrastructures. Past experimentations have shown the need as well as usefulness of such system. Before Cloud2IR an initially laboratory oriented system was used. Based on such system Cloud2IR has benefited of the experimental knowledge acquired to redefine a lighter architecture using generics standards, more appropriated to autonomous operations on field and which can be later included in a wide distributed architecture. The system has been developed in order to cut down software integration time which facilitates the system adaptation to each experiment specificity. That's why we propose a bi- headed architecture. A specialized part, it represents the sensor specific development as well as their drivers and their different fixed configurations. In our case, as infrared camera are slightly different than other kind of sensors, the system implement in addition an RTSP server which can be used to set up the FOV as well as other measurement parameter considerations and a generic part, which can be seen as the data management side. This last part can be seen as the first embryo of a future generic framework dedicated to the data management of local multisensors (DaMaLoS). It's able to aggregate any sensor data, type or size and automatically encapsulate them in various generic data format as HDF5 or cloud data as OGC SWE standard. This whole part is also responsible of the acquisition scenario the local storage management and the network management through SFTP or SOAP for OGC web services. The data side only need an XML configuration file and if a configuration change occurs in time the system is automatically restarted with the new value. Cloud2IR has been deployed on field since more than one year at the SenseCity outdoor test bed and several month at the Inria test bed, both located in France. The system aggregates various sensors as infrared camera, a GPS, multiple pyranometers, a weather station and a proprietary access to the SenseCity data viewer. The SenseCity test bed consists of two lodges, both are instrumented and one is monitored by infrared, in this last lodge various insulating defects have been included in order to test our systems and models. This paper will show the results of the first year of the system use.
\end{abstract}

\section{Introduction}

Following research works initiated in the European project ISTIMES [1], the project Cloud2SM (Cloud architecture design for Structural Monitoring with in-line Sensors and Models tasking) [2], has been launched to develop a robust information system able to assess the long term monitoring of civil engineering structures as well as interfacing various sensors and data. The specificity of such architecture is to be based on the notion of data processing through physical or statistical models. Thus the data processing, whether material or mathematical, can be seen here as a resource of the main architecture. The project can be divided in various items as the sensors and their measurement process, the storage resources, the computational resources, the models themselves, the user interface, and the structures themselves. The project intend to be standardized by following the OGC recommendations for its web interfaces and for example the hdf5 for its local data format. Figure 1 presents a global schematic view of Cloud2SM project.

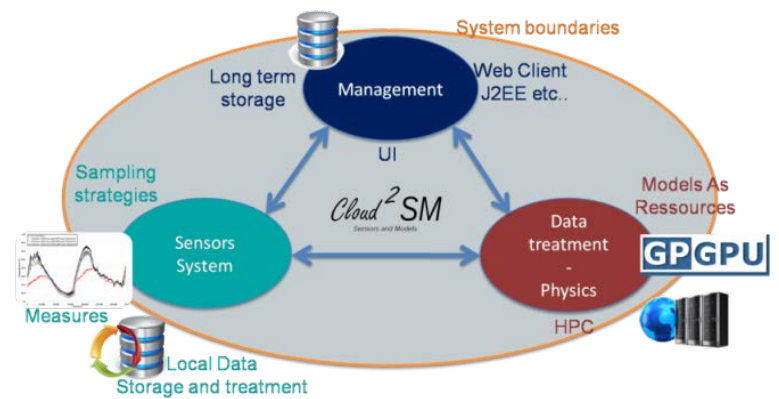

Fig. 1 Cloud2SM architecture principle data side.

Cloud2IR represent the infrared and environmental sensor side of Cloud2SM project, i.e. its connection to the 


\subsection{1/qirt.2016.028}

In this paper we first propose a short review of the standards implemented in our project. Then the design and development of Cloud2IR are presented and discussed. Two deployments on real test site are presented and results obtained discussed. Finally, conclusion and perspectives are proposed.

\section{Overview of the used standards}

\subsection{Genicam standard for infrared sensors}

The Generic Interface for Cameras (GenICam) is a generic programming interface for machine vision (industrial) cameras. The goal of the standard is to decouple industrial camera interfaces technology, such as GigE Vision or Camera Link etc., from the user application programming interface (API). GenICam is administered by the European Machine Vision Association (EMVA) [3].

GenICam works by utilising a camera description file in the form of an XML Schema file which is easily interpreted by GenICam, as shown in figure 2. The file is located on the camera and when connected communicates its available features to the software automatically. At either compile time or run time, GenICam interprets the camera description file to generate a $\mathrm{C}++\mathrm{API}$ or allow the user to enumerate the features found in the file to handle in a generic fashion, such as displaying them in a GUI.

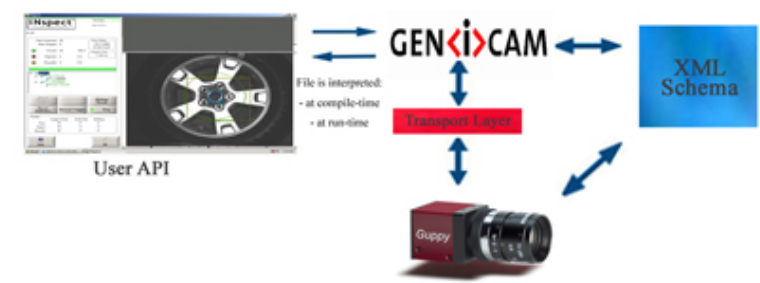

Fig. 2 GenICam principle schematic illustration

The fact is that a growing number of infrared camera manufacturer replace their proprietary solution by a GenICam implementation [4] which allow us to define lighter drivers through the aravis library to get data and send setup request to the infrared camera [5].

\subsection{Data standardisation}

In this world where a lot of actors (industrial, academics, public policy...) produce and aggregate massive data flows, the data standardisation is a key to insure the interoperability between systems. A growing number of solutions are developed to give scientists an access to those data and to computing systems [6-7]. From that point of view raw data exist into various format (matlab, csv, text, binary, proprietary...), those are called legacy data and have to be a posteriori shaped into intelligible format (hdf, netCFD, standard DB, O\&M encodings, postGIS...). Here we present standards used in cloud2IR in order to cut off the conversion operations. The main objective of this development action plan is, at term, to fully interoperable in term of data as well as metadata [8-9].

\subsubsection{Hdf5}

Firstly proposed by the National Center for Supercomputing Applications and now developed by the HDF Group, the Hierarchical Data Format is a generic data container able to structure and describe a huge amount of data. Aviable in open source (BSD) the HDF library is common to various systems and computing languages (Matlab, C/C++, Java, Python). Various versions of the format have been proposed, the Hdf5 (the last one) simplify the structure as the access strategies to the data contained on the file, from that version it exist now only two entities implemented on the standard completed by their metadata:

- The datasets which contains the data themselves whatever their size, dimension or type as those are externally defined.

- The groups in which can be stored dataset or other groups.

- The metadata which are the key to a well described file which can be used by a third party.

The figure 3 summarizes the HDF5 architecture. 


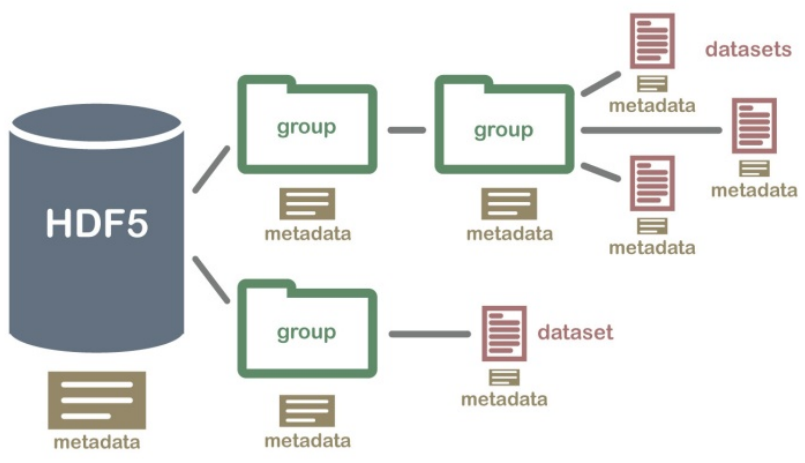

Fig. 3 HDFfile format hierarchy

This structure is equivalent to the file system of an Operating System and presents the same access philosophy.

For us this file format present at least three main advantages, the external typing and sizing of the data which allow the auto-generation of dataset, the tree structure which facilitate the data access and the metadata management which enforce the interoperability with other systems, note also that the HDF5 natively provide compression algorithm which can be useful on intensive use of IR camera. The HDF5 container can be contained itself on a net-CDF format which is another highly used standard for raw data, for example purpose such file can be directly send and use by most of the HPC (High Performance Computer/Computing) centers, which comfort us in our choice. Once our system is able to produce standardized raw data it will be able to interact with other ISO compliant solution as the OGC sensor observation and measurement.

\subsubsection{OGC observations and measurement (SOS)}

First let's introduce the OGC. The Open Geospatial Consortium (OGC), an international voluntary consensus standards organization, originated in 1994. In the OGC, more than 500 commercial, governmental, nonprofits and research organizations worldwide collaborate in a consensus process encouraging development and implementation of open standards for geospatial content and services, GIS data processing and data sharing. One of the main goal of this consortium is to promote open standards able to create wide information systems. The figure 4 left show that the OGC act as a link between the accepted standards like ISO and the information technologies as the World Wide Web Consortium (W3C). The OGC provide various standards, from the sensor side the main paradigm is resumed by the Sensor Web Enablement (SWE) see figure 4 right. The SWE standards enable developers to make all types of sensors, transducers and sensor data repositories discoverable, accessible and useable via the Web.
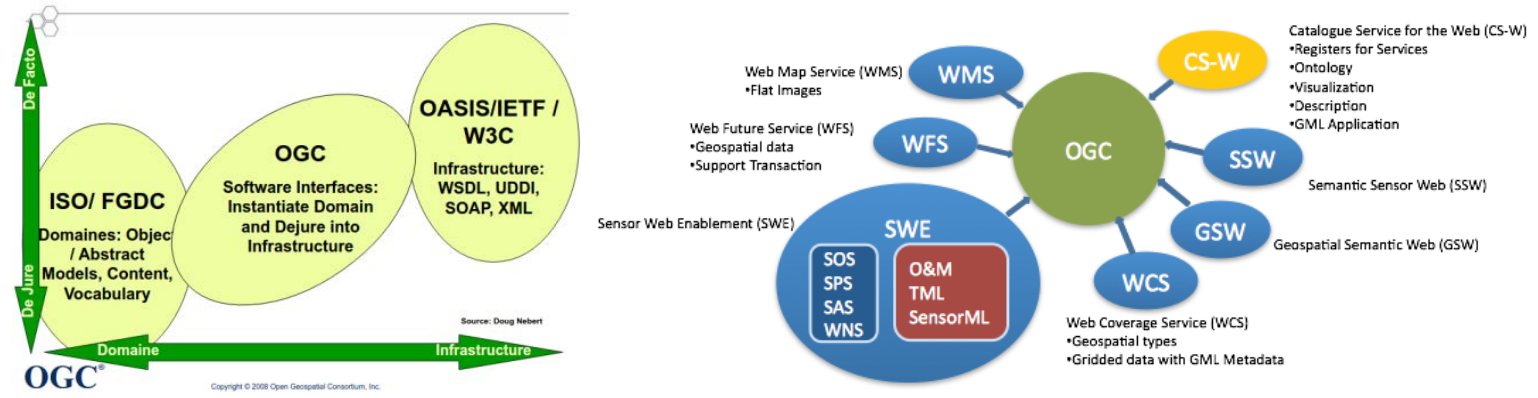

Fig. 4 OGC standards schematic view

The SWE can be divided in two essential components; first the encodings (O\&M, sensorML etc...) which are made to represents data, sensors and their metadata in XML format on the second hand the SWE provide various web services like the Sensor Observation Service (SOS) which allow real-time query of sensor data and sensor data time series all described by the encodings presented bellow [10].

It can be seen that the OGC standards are mainly based on web services idea, which beside their apparent complexity allows a generic use of their proposed resources, in the SOS case the measurements and the sensor description itself.

\subsubsection{Discussion on the used standards}

Our initiative in this project is to provide sensors architectures and framework able to produce standardized data. The HDF5 allow us to store locally and externally a huge amount of data, for that point the file format is well adapted to the thermal long term monitoring of civil engineering structures. The other point is that if the HDF5 file is well-informed with the 


\subsection{1/qirt.2016.028}

right metadata following the ISO recommendations it can be easily converted into a cloud information system based on the OGC-SOS web service. The fact is that at term our system will be able to produce in the same time "stone" data (HDF5 ) which can be send through a network (step 1 figure 5) and cloud data (SOS) and provide redundancy of critical data, but as civil engineering structure is not always connected to the network we concentrate our development into HDF5 integration then externally converted into SOS web format (step 2 figure 5) before to develop a real sensor web interface (step 3 figure 5).

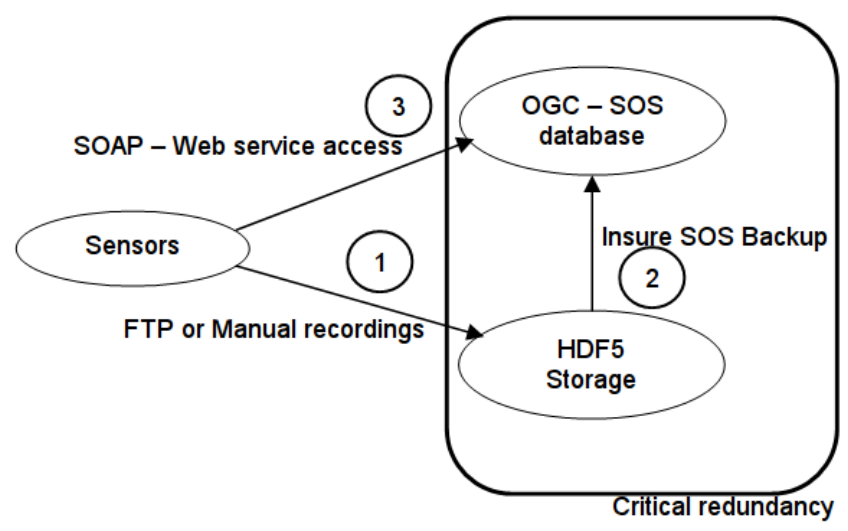

Fig. 5 System future integration

\section{Cloud2IR Development}

Cloud2IR represent the sensors system used inside the Cloud2SM project.

\subsection{Genesis and design brief}

For many years now our team have been uses a complete system called IrLaw to control IR cameras and various environmental sensors [11-12]. IrLaW have been built firstly for local experiment and on a legacy ubuntu, over time this system shows its limits for a long term easy use. Two of them was problematic for us, first the HMI (Human Machine Interface) which consume computing and power resources, secondly the system needed an high development cost for maintenance operation and adaptation to each experiment specificity. So inside the Cloud2SM project it have been decided to develop a system which can answers four main item:

- $\quad$ Reusing part of the IrLaW software and manage Technical debt

- $\quad$ No HMI

- $\quad$ Provide a generic development framework to adapt effortlessly to the system to each experiment

- Insure the data standardisation

One of the best parts of IrLaW was its way to manage sensors. Real time sensors, as the IR camera, were directly controlled inside the system core thanks to a circular buffer, the non real time or sub sampled sensors was them demonized by a specific service (call serviced) and their data where stored on a register base accessible by both IrLaW and the sensors themself. For cloud2IR those both sensors management where conserved, and the main system has been demonized, figure 6 .

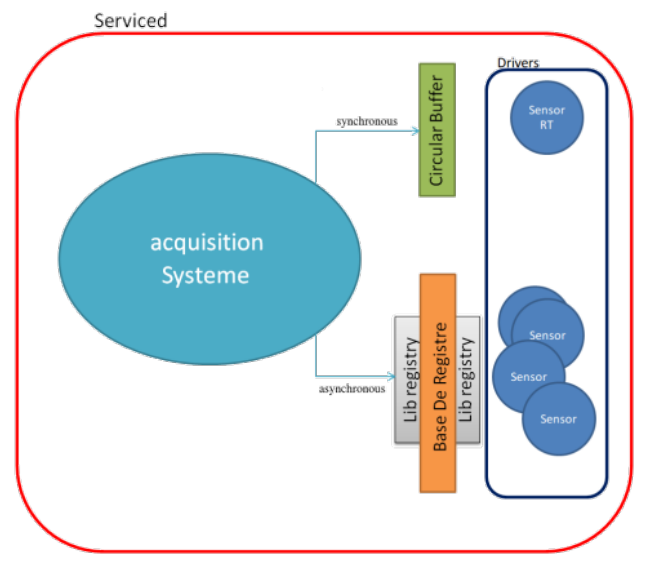

Fig. 6 From IrLaW to Cloud2IR 


\subsection{Modularity}

As it have been presented the modularity of the system is the key to adaptation so it has been developed in order to cut down software integration time which facilitates the system configuration to each experiment specificity. We propose a bi- headed architecture with a specialized part which represents the sensor specific development as well as their drivers and their different fixed configurations and a generic part, which can be seen as the data management side.

\subsubsection{DaMaLoS}

DaMaLos stands for Data Management of Local multiSensors, it correspond to the generic part of the system. Based on the HDF5 external data typing (see below), this module can be seen as a generic framework to manage data produced by the sensors. It instantiate through a generic interface all the sensors and control the data access loop (not the requesting). This side of the system is weakly coupled with the sensor side. It can be seen as a general framework able to aggregate any sensor data, type or size and automatically encapsulate them in various generic data format as HDF5 or cloud data as OGC SWE standard. This whole part is also responsible of the acquisition scenario the local storage management and the network management through SFTP or SOAP for the OGC frame. This can be resume in various functionalities:

- The Sensor interface: this interface is the entering point of the sensors on the framework, each sensor attach their bytes data and specify theirs type, size and need of compression.

- The data standardisation, first in HDF5 file format and in the future we will implement the OGC O\&M.

- The local data management: Users can specify the amount of data they want on local backup

- Network management: The framework manages all the networking operations.

- The acquisition strategy : For now the system can only subsample data

The whole configuration is achieved by an XML file.

\subsubsection{Addressing experiments specific instrumentation requirements}

For each experiment we have different sensors and different need, as the data management is already achieved by DaMaLoS the development effort is then concentrated on the sensor driver if it's not already build. The resources can now be oriented to specific treatment as computing algorithm or for the IR camera the redirection of their video flux to a streaming server (RTSP). Once each driver is attach to DaMaLoS through the sensor interface (one code line) the data management is automatically done and hdf5 file are directly shaped.

\subsection{Typical custom design for outdoor experiments}

Let's present a typical custom implementation of the system designed for an outdoor long term experiments. Typically, the system implements a weather station, pyranometer, a GPS and an uncooled IR Cam using GiGe Genicam standard and the synchronous update strategy inherited from IrLaW.

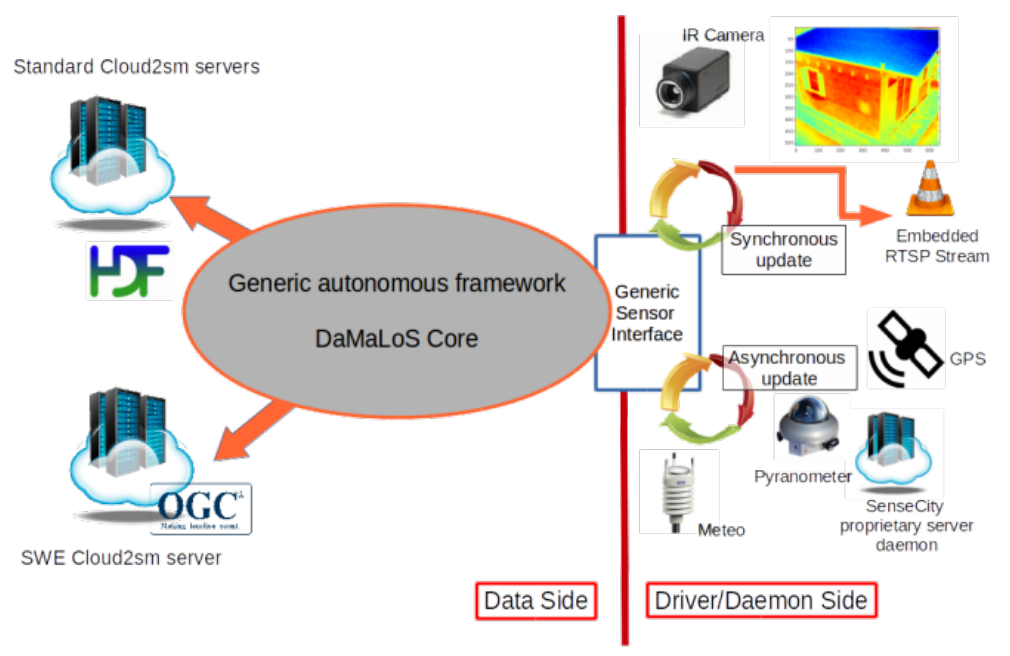

Fig. 7 SenseCity implementation 


\subsection{1/qirt.2016.028}

The figure 7 present the architecture, as the data management does not need specific development resources we were able to focus on the driver development and build an embedded RTSP system (Real Time Streaming Protocol) to access to the infrared stream without disturbing the acquisition process. Another point is that we connect to our register database a light TCP/IP client which can also provide a part of our data to an external proprietary server (different from the Cloud2SM one). Those both new functionality reveal our former modularity needs.

\section{Test Beds and results}

\subsection{The Sense-City experimental test site}

The Sense-City [13] Equipex (see Figure 8 (a)) is a large scale equipment which has received support from the French government in the framework of the "Programme d'Investissement d'Avenir" (Future Investment Programme). This project is led by the French Institute of Science and Technology for Transport, Development and Networks (IFSTTAR) with ESIEE-Paris, the Laboratory of Physics of Interfaces and Thin Films (LPICM) and the Scientific and Technical Centre for Building (CSTB) as consortium partners. The Sense-City project is a suite of high-quality facilities for the design, the prototyping and the evaluation of performance and risks of innovative micro- and nanotechnology based sensors devoted to measurements in urban environment. At this date the cornerstone of the mini-city has been posed and the Equipex will be fully operational in 2017.

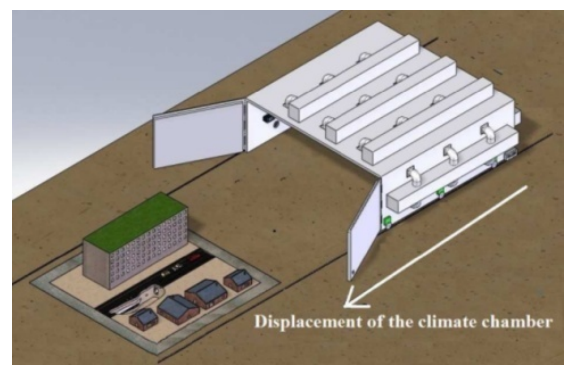

(a)

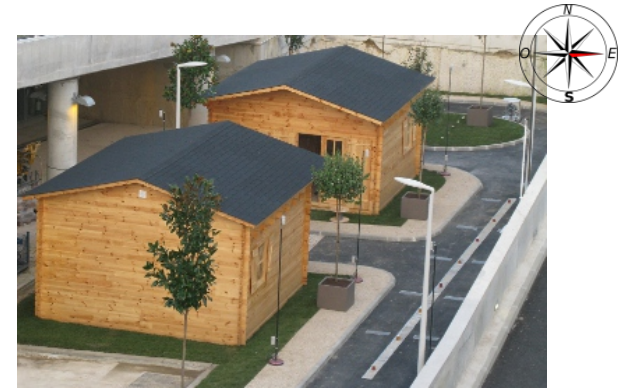

(b)

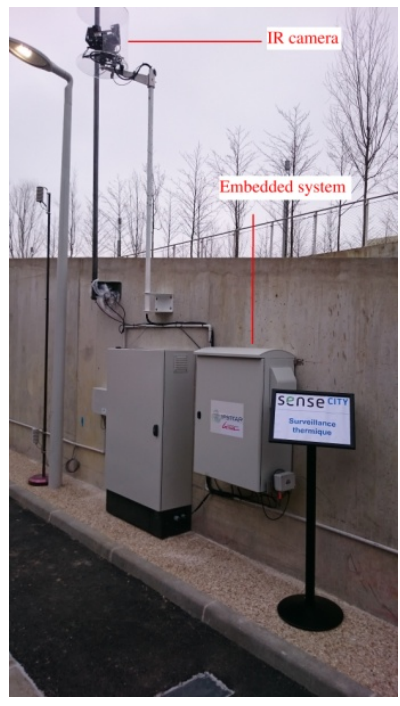

(c)

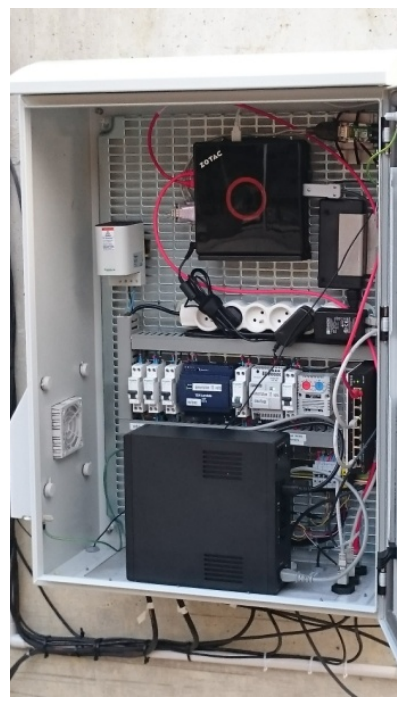

(d)

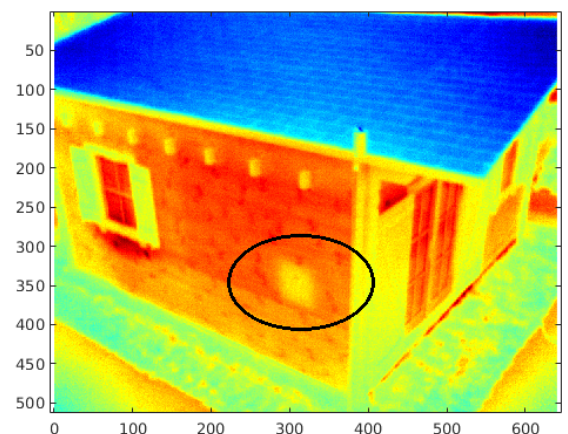

(e)

Fig. 8 Future Sense-city test site (a) actual first demonstrator (b) deployed system (c) hardware system (d) thermal image acquired at sunrise (e)

While waiting the full site availability a first demonstrator have been built at the IFSTTAR headquarter office (figure $8 \mathrm{~b}$ ). This first site is fully instrumented (smart road, energy consumption efficiency, GPR test site...) and this is where our long term infrared monitoring system has been deployed since over a year now (figure $8 \mathrm{c}$ and d). The IT system is deployed on a zotac mini PC under ubuntu. Composed of an IR camera, a weather station a pyranometer and a GPS 
the system is designed to operate on normal or emergency power supply. The figure 8 e shows a sample of thermal imaging acquired by the system.

On figure 9 and 10 we present the time evolution of four characteristics pixels over a week and also the air temperature and solar heat flux evolution with time. The four pixel are respectively located on the front wall (left wall with a windows on the thermal image), on the insulating defect (visible on figure 7e), on the roof and on the opposite side of the lodge.
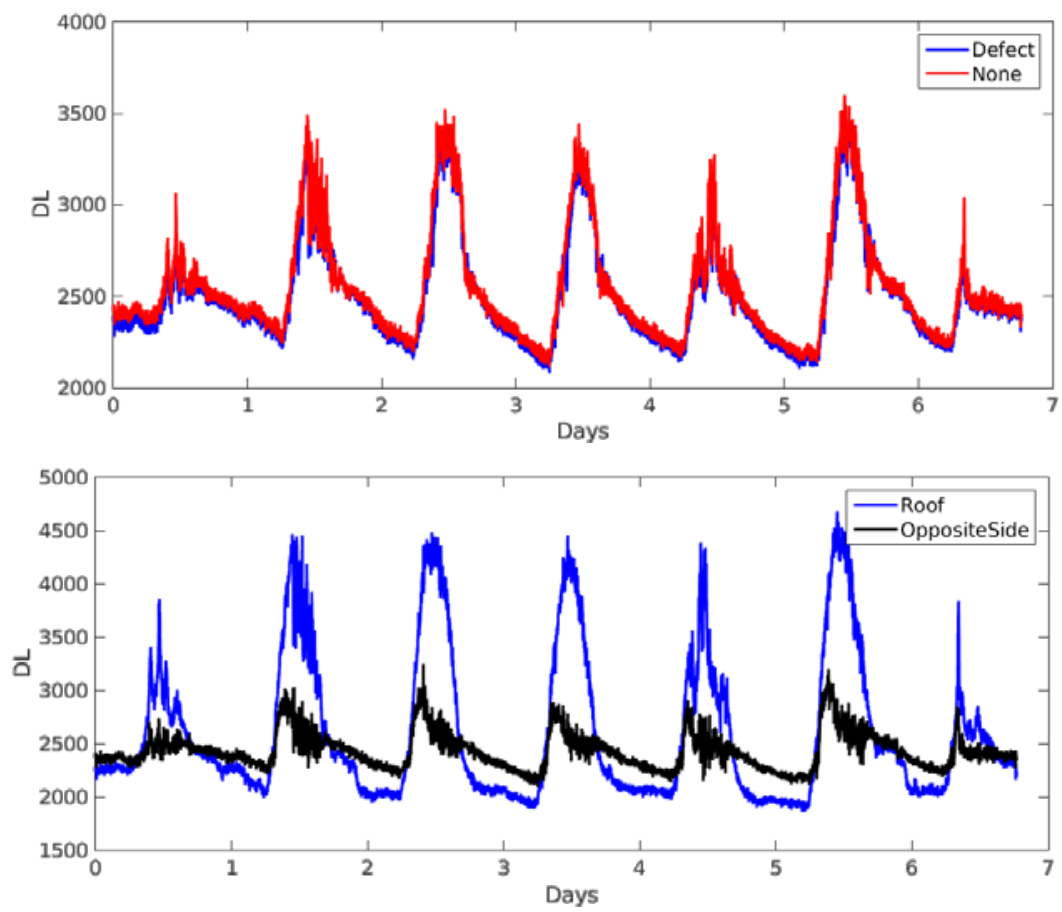

Fig. 9 Evolution of four points from the 10 to the 16 march 2016
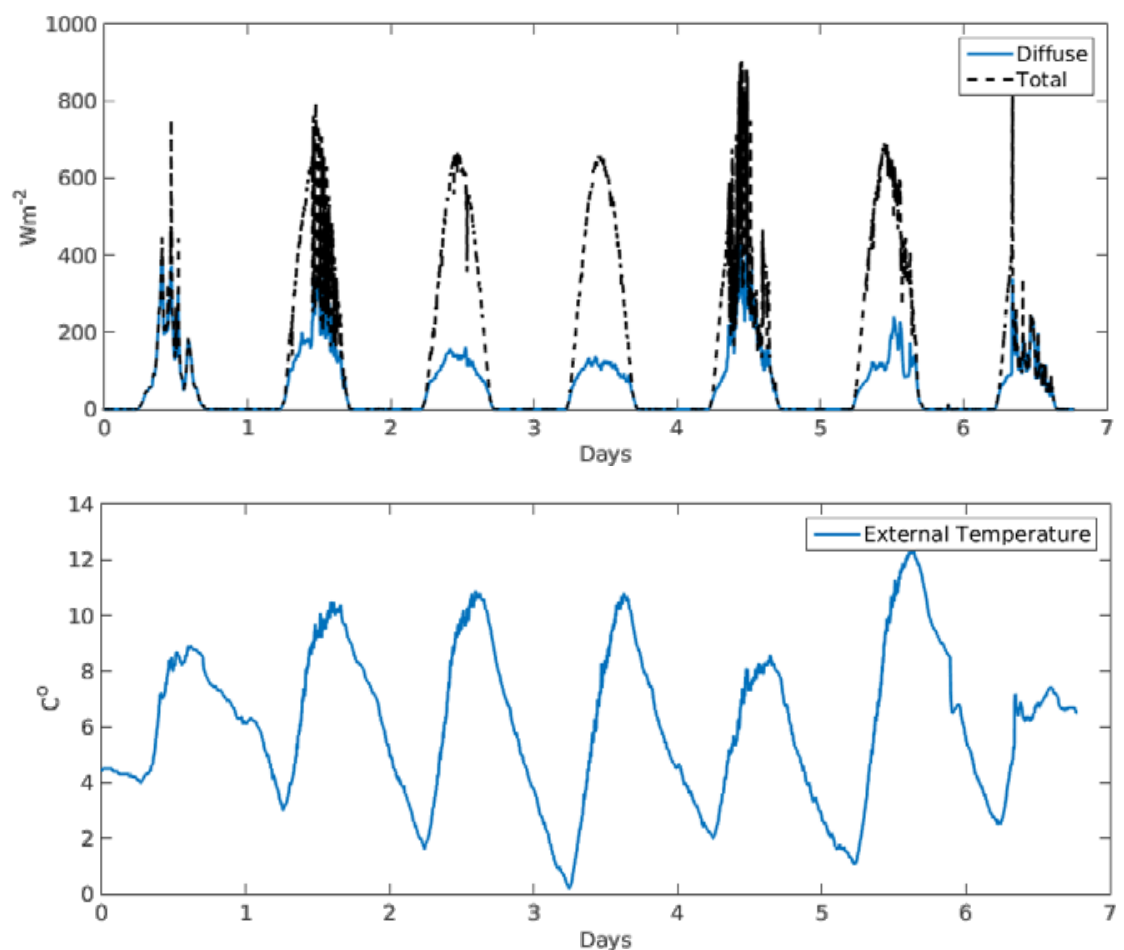

Fig. 10 Evolution of solar heat flux and external temperature from the 10 to the 16 march 2016

Over this seven days it appears that the insulating flaw have a slight effect (above the ambient noise) mainly visible on the relaxation phases, especially when the weather seems cloudy. As the lodge is east oriented, the oppose side 


\subsection{1/qirt.2016.028}

is sunbathed once a day at sunrise and during few hours, whereupon the opposite side enter in relaxation phase. As the first observation showed and the orientation of the lodge it will certainly be interesting to induce another insulating defect on the opposite side. As regards the roof, it show a stronger behaviour, its solar orientation and the fact that the inner roof is thermally insulated can induce such attitude.

\subsection{Inria office buildings test site}

A second test site have been instrumented in Inria headquarter at Rennes France. This test bed differ from the first one as the building currently in use, present various face with different orientation and composed of multi-materials, see figure 11 . Those particularities make this test bed unique and a promising site for future experiments.

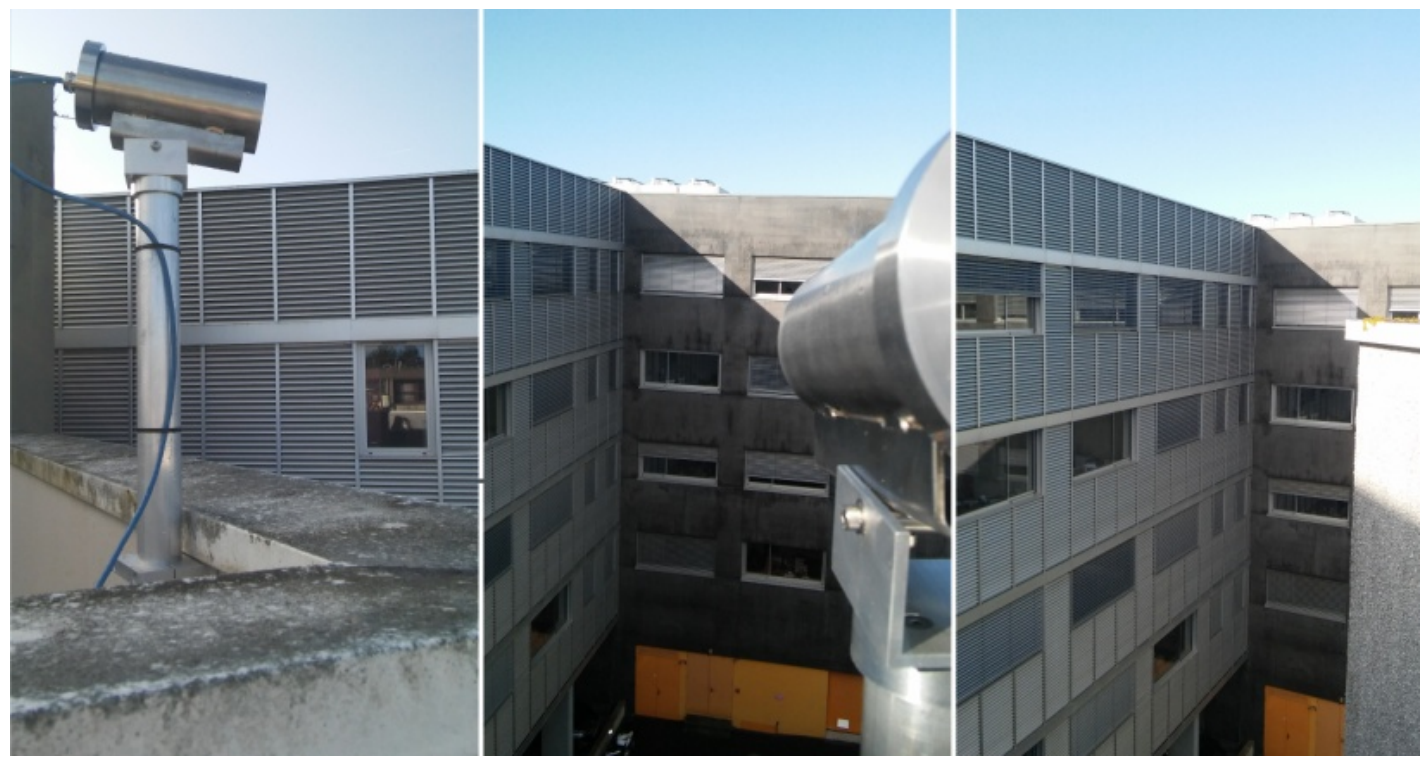

Fig. 11 Inria test site

For now the site is composed of just an uncooled IR camera and run since the beginning of 2016. Owing to it's particularity the only data which will be presented here is a composite image, figure 12.
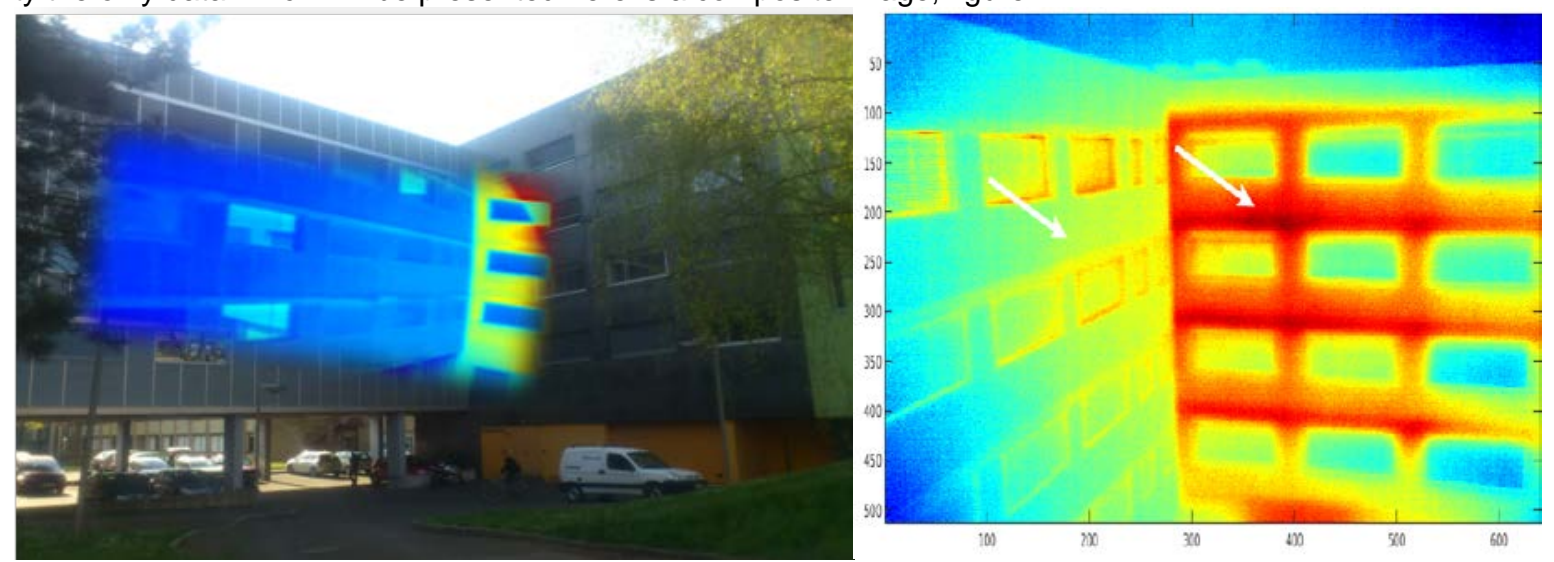

Fig. 12Composite image of the Inria test bed, and main IR image

The figure 13 shows the evolution of two pixels belongings to each buildings (see arrows figure 11). Both pixels are East oriented so they are sunbathed at least in the morning, but (as for the lodges on the senseCity test site) the buildings oriented north east have a smaller solar contribution the rest of the day. Another point is that the third and fourth day, the S-E buildings thermal evolution seems particularity eroded by something, only its amplitude appears perturbed. 


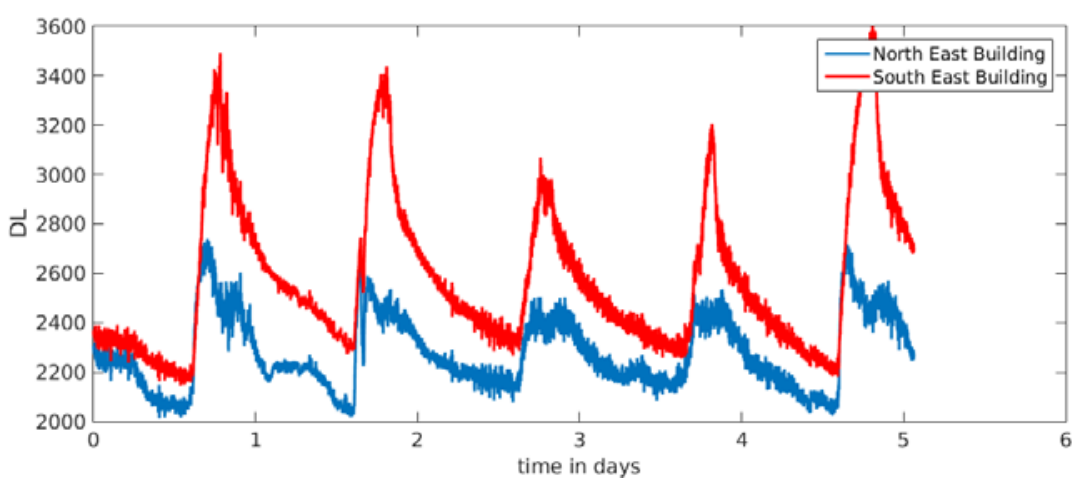

Fig. 13 Digital levels evolution of two pixels from the 25 to the 29 February 2016

The figure 14 present some environmental variable measured at the Rennes airport (about 10Km from the test site), Theses data have been obtained thanks to http://www.infoclimat.fr/. By looking the 27 and 28 February, the external temperature and humidity does not appear to have a different behaviours than the other days. However the wind speed increase and the wind orientation is about zero degrees $\left(0^{\circ}\right.$ and $360^{\circ}$ correspond to the North) and does not change for a while, by crossing the available data it appear that the south east oriented building face a strong wind the 27 and 28 February affecting its surface thermal amplitude.
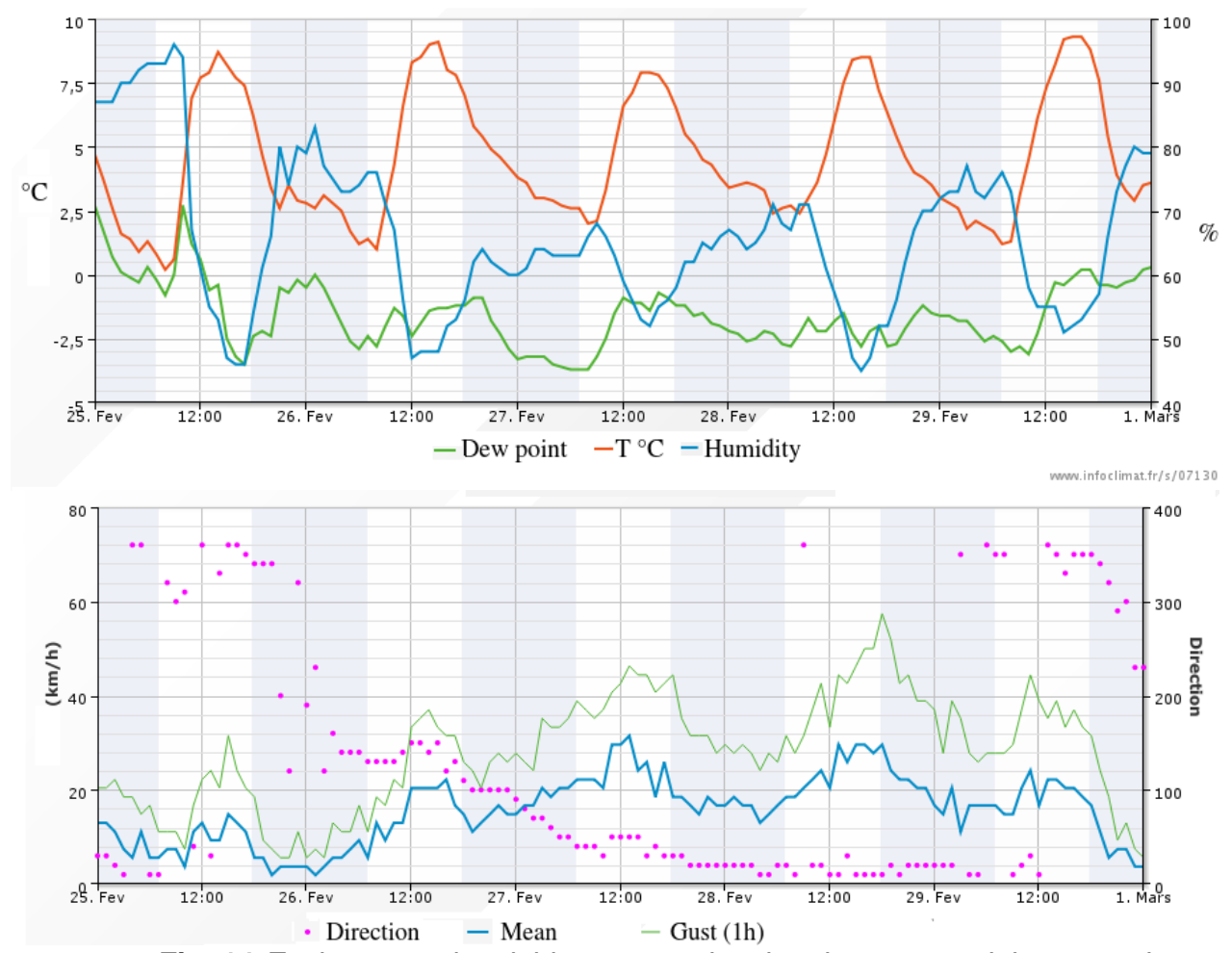

Fig. 14 Environmental variable measured at the airport around the same dates

Complementary instrumentation will be installed on this second test site, in order to develop quantitative analysis.

\section{Conclusion and perspectives}

In this paper a new software architecture "Cloud2IR", able to assess the thermal long term monitoring of civil engineering structures, was presented. This software natively implements data standardisation and allow engineers and researchers to focus on the experimental specific development thanks to the DaMaLoS core which support all the data shaping operations and storage as well as the networking. A specific development has been done to build an embedded real time streaming server for the Genicam infrared camera. The robustness of this new architecture and sensors are still under evaluation on 2 test sites, and on near future on a third one (real bridge). 


\subsection{1/qirt.2016.028}

Anyway, thanks to the large data set acquired on the Sence-City test site and the measurement of environmental parameter at each time step of thermal image acquisition, investigation on an adapted lockin approach will be initiated. Statistical approach could also be investigated on such large data set. Finally, we also have the opportunity on this test site to follow the ageing of some building material.

The second test site allows us to test IR long term monitoring on a building with a higher thermal inertia tha the Sence-City lodges. The configuration of this second test site is also helpful to develop radiative correction models in presence of a non-negligible reflective object in the scene.

For both experiments, it is planned to make cross-analysis with local sensor that are or will be added to the buildings in a near future.

\section{REFERENCES}

[1] M. Proto et al., « Transport Infrastructure surveillance and Monitoring by Electromagnetic Sensing: the ISTIMES project », Journal Sensors, Sensors 2010, 10(12), 10620-10639; doi:10.3390/s101210620, December 2010.

[2] Antoine Crinière, Jean Dumoulin, Laurent Mevel, Guillermo Andrade-Barosso, Matthieu Simonin. The Cloud2SM Project. European Geosciences Union General Assembly (EGU2015), Apr 2015, Vienne, Austria. 2015. <hal$01144162>$

[3] http://www.emva.org/standards-technology/genicam/ (20/04/2016)

[4] Technical report : GenICam ICD FLIR AX5 Camera - PC 19/02/2013

[5] https://wiki.gnome.org/Projects/Aravis (20/04/2016)

[6] L. Wyborn and B. Evans "The Big Challenge in Big Earth Science Data: Maturing to Transdisciplinary Data Platforms that are Relevant to Government, Research and Industry" EGU2016-11686-1, 2016 EGU General Assembly 2016

[7] A. Ip et al. "Unleashing Geophysics Data with Modern Formats and Services" EGU2016-11686-1, 2016 EGU General Assembly 2016

[8] ISO 19156:2011 Geographic information -- Observations and measurements

[9] V. Choi and M. Folk "Investigations into using HDF5 for product model data -B-Spline and Cartesian Point data in HDF5", The HDF Group, 2007

[10] OGC, "Sensor Observation Service Interface Standard", OGC 12-006, 2012.

[11] J. Dumoulin et R. Averty, « Development of an infrared system coupled with a weather station for real time atmospheric corrections using GPU computing: Application to bridge monitoring", QIRT 2012 (11th International Conference on Quantitative InfraRed Thermography), Naples, Italie, Juin 2012.

[12] J. Dumoulin and R. Averty "Infrared imaging system monitors transportation structures in real time" 23 January 2014, SPIE Newsroom. DOI: 10.1117/2.1201401.005063

[13] F Derkx, B Lebental, T Bourouina, Frédéric B, C Cojocaru, and al..The Sense-City project.XVIIIth Symposium on Vibrations, Shocks and Noise, Jul 2012, France. 9p, 2012.<hal-00860842> 